\title{
Eléments nouveaux dans le calcul des cycles des usines marémotrices
}

\section{A new approach to tidal power plant calculations}

\author{
PAR M. GAUTHIER,
}

INGÉNIEUR A LA SO.GR.E.A.H., GRENOBLE

\begin{abstract}
La détermination de la marche optimale d'une usine marémotrice est un problème complexe de calcul des variations. Bien que les conditions locales d'extremum fussent connues, les problèmes liés aux changements de types de fonctionnement de l'usine (mise en route ou arrêt des turbines, des vannes...) n'étaient pas complètement éclaircis.

Leur étude systêmatique, dont nous présentons les résultats, a permis d'aborder de façon plus stricte les problèmes d'optimum global.
\end{abstract}

\begin{abstract}
The determination of optimum tidal power plant operating conditions is a complex variation calculation problem. Although the extreme local conditions are known, the various problems associated with changeovers from one operational condition to another still needed clarifying (e.g. starting and stopping turbines, gate operation, etc.).

Thanles to a systematic investigation of these problems, a stricter approach can now be made to overall optimum operation cases than before. The results of this investigation are presented in the article.
\end{abstract}

\section{INTRODUCTION}

Une usine marémotrice peut adopter différents types de fonctionnement que nous notons :

0 arrêt;

turbine directe

1 ou pompe inverse;

1 , turbine directe

ou pompe inverse; et vannage;

2 turbine inverse

fou pompe directe;

2' $\quad$ turbine inverse

$\left\{\begin{array}{l}\text { ou pompe directe; }\end{array}\right.$
Les conditions d'optimum de fonctionnement pour chaque type ont été étudiées en détail par M. Gibrat. Mais le problème de l'optimum global se heurtait encore à de nombreuses difficultés. En effet, ce n'est pas un problème de calcul des variations classiques, car à chaque instant nous avons le choix entre plusieurs types de fonctionnement.

Nous avons étudié l'influence d'un tarif variable en fonction de l'heure et de la puissance produite sur les conditions de changement de fonctionnement de l'usine. 


\section{I. - MISE EN EQUATION SINGULARITÉ DU PROBLEME}

\section{Fonctions inconnues et paramètres.}

Nous supposerons l'usine équipée de groupes identiques, tous les groupes non arrêtés fonctionnant dans les mêmes conditions. Les principales grandeurs liées au problème sont:

$$
\begin{aligned}
h(t) & \text { Cote de la marée; } \\
z(t) & \text { Cote de la retenue; } \\
\mathrm{H}(t) & \text { Hauteur de la chute; } \\
\mathrm{Q}(t) & \text { Débit total; } \\
\Sigma(z) & \begin{array}{l}
\text { Surface de la retenue à la } \\
\text { cote } z ;
\end{array} \\
n & \text { Nombre de turbines en fonc- } \\
& \text { tion; } \\
\mathrm{N}(q, \mathrm{H}) & \text { Colline turbine; } \\
\mathrm{V}_{0}(h, z) & \text { Débit maximal des vannes; } \\
\mathrm{V}(t) & \text { Débit des vannes; } \\
p_{1}(t, \mathrm{~N}) & \text { Tarif marginal; } \\
\mathrm{P}_{1}(t, \mathrm{~N}) & \text { Tarif; } \\
\mathrm{U}\left(t_{1}, t_{2}\right) & \text { Recette globale de l'instant } t_{1} \\
& \text { à l'instant } t_{2} .
\end{aligned}
$$

Six de ces grandeurs $(1,5,7,8,10$ et 11) sont des paramètres liés à la construction de l'ouvrage et aux conditions économiques de son exploitation; par définition :

$$
\mathrm{P}_{1}(t, \mathrm{~N})=\int_{0}^{\mathrm{N}} p_{1}(t, x) d x
$$

Les six autres grandeurs sont liées par les trois relations fonctionnelles :

I

$$
\begin{aligned}
\mathrm{H} & =z-h \\
\mathrm{Q} & =-\dot{z} \Sigma_{z}^{\prime}(z) \\
\mathrm{U}\left(t_{1} t_{2}\right) & =\int_{t_{1}}^{t_{z}} \mathrm{P}(t, n \mathrm{~N}) d t
\end{aligned}
$$

Le problème posé revient donc à la détermination de trois fonctions inconnues par la condition que l'intégrale (I) soit maximale.

\section{Grandeurs réduites.}

Le nombre maximal de turbines est fixé par construction; soit $n_{\mathrm{M}}$.
Il a été commode de rapporter toutes les grandeurs à une turbine. Nous poserons pour cela :

$$
\begin{aligned}
& \lambda \quad=n / n_{\mathrm{M}} \\
& \mathrm{S}(z)=\Sigma(z) / n_{\mathrm{M}} \quad \text { Surface de l'es- } \\
& q \quad=(\mathrm{Q}-\mathrm{V}) / n_{\mathrm{M}} \stackrel{\text { tuaire; }}{\text { Débit des }} \text { rapportés }
\end{aligned}
$$

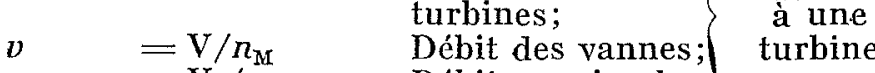

$$
\begin{aligned}
& v_{0}=\mathrm{V}_{0} / n_{\mathrm{MI}} \quad \text { Débit maximal } \\
& \text { u. } \quad=\mathrm{V} / \mathrm{V}_{0} \\
& \text { des vannes; }
\end{aligned}
$$

Nous avons utilisé comme fonction inconnues principales :

$$
z(t) \quad \mu(t) \quad \lambda(t)
$$

D'autre part, nous admettons, ce qui est pratiquement vrai, que $\lambda$ varie de façon continue.

\section{Equations du problème.}

$q$ et $\mathrm{H}$ s'expriment en fonction de $z, \dot{z}, \mu$ et des paramètres :

$$
\begin{aligned}
\mathrm{H} & =z-h \\
q & =-\dot{\mathrm{S}}-\mu v_{0}
\end{aligned}
$$

L'intégrale I s'exprime donc en fonction de $z$, $z, \lambda$, ss et des paramètres :

$$
\text { II } \quad \mathrm{U}=\int_{t_{1}}^{t_{2}} \mathrm{P}\left[t, \lambda \mathrm{N}\left(\frac{q}{i}, \mathrm{H}\right)\right] d t
$$

Nous allons étudier la famille d'extrémales issues d'un point donné, c'est-à-dire qu'à un instant donné nous supposerons donnés :

$$
z, \lambda \text { et } \mu
$$

et nous nous proposons d'étudier :

- Si, par tout point, passent une ou plusieurs extrémales du problème;

- De combien de paramètres elles dépendent;

- Une extrémale étant choisie, peut-on toujours la prolonger? 
Une condition nécessaire pour qu'il existe une solution continue et à dérivée première continue, intérieure à son domaine de définition, est que, pour tout $\mathrm{T}$ :

$\operatorname{III}\left\{\begin{aligned} \frac{\partial \mathrm{P}}{\partial \dot{\mathrm{H}}} & =\int_{t_{1}}^{\mathrm{T}} \frac{\partial \mathrm{P}}{\partial \mu}[t, \lambda(t), \mu(t), z(t), \dot{z}(t)] d t \\ & + \text { constante }\end{aligned}\right.$
Or, puisque $\mathrm{P}$ n'est pas fonction explicite de p. et de $\lambda$, les équations différentielles (1) et (2) se réduisent à :

$$
\frac{\partial \mathrm{P}}{\partial \mu}=0 \quad \text { et } \frac{\partial \mathrm{P}}{\partial \lambda}=0
$$

résolvables sans intégration.

S'il existe des courbes extrémales issues d'un point donné, elles dépendront donc au plus d'un seul paramètre. En particulier, il n'y a pas en général d'extrémale joignant deux points quelconques (1).

\section{II. - VANNAGE}

\section{Débit des vannes.}

La condition III (1) se développe immédiatement en :

$$
-v_{0}(t) p(t, \mathrm{~N}) \frac{\partial \mathrm{N}(q / \lambda, \mathrm{H})}{\partial(q / \lambda)}=0
$$

ce qui est visiblement irréalisable. Le problène n'admet donc pas de solution continue en $\mu$ intérieure à son domaine de définition. Les seules marches acceptables sont donc:

$$
\mu=0 \quad \text { ou } \mu=1
$$

c'est-à-dire que le débit des vannes ne peut être que nul ou maximal, quel que soit le tarif. Ce résultat acquis par M. Gibrat lorsque le tarif varie en fonction du temps seulement, était évidemment prévisible.

On démontre d'autre part sans difficulté que, pour une loi de débit donnée $Q(t)$,

$$
\text { si } \frac{\partial \mathrm{N}}{\partial q}\left(\mathbf{Q} / n_{\mathbb{M}}, \mathrm{H}\right)>0
$$

tout vannage diminue la recette, donc $\mu=0$;

$$
\text { si } \frac{\partial \mathrm{N}}{\partial q}\left(\mathrm{Q} / n_{\mathrm{M}}, \mathrm{H}\right)<\mathbf{0}
$$

il existe un vannage améliorant la recette. Il faut donc vanner, et seul le débit maximal est permis : $\mu=1$.

La frontière entre ces deux domaines est la ligne de puissance maximale.

\section{Mise en route des vannes. - Réfraction de l'extrémale.}

Cie sont les changements de fonctionnement (1) - $\left(1^{\prime}\right)$ et $(2)-\left(2^{\prime}\right)$. Soit une extrémale issue d'un point de la colline turbine où :

$$
\frac{\partial \mathrm{N}}{\partial q}>0
$$

c'est-à-dire où nous sommes en marche (1), et supposons que l'extrémale rencontre en $\mathrm{M}$ à l'instant $t_{1}$ et à la cote $z_{1}$ la ligne de puissance maximale. D'après ce que nous venons de dire pour $t<t_{1}$, le prolongement de l'extrémale sans vannage n'est pas optimal.

Peut-on débuter par la marche $\left(1^{\prime}\right)$ ?

Le débit des turbines est continu, donc le nouveau débit total est parfaitement déterminé :

$$
\mathbf{Q}=n_{\mathrm{M}}\left[q\left(t_{1}\right)+v_{0}(\mathrm{H}, h)\right]
$$

cela détermine $\dot{z}(t)$ pour $t=t_{1}+0$.

Nous verrons dans la troisième partie qu'en un tel point, $\lambda=1$ et que, par suite, nous disposons de tous les éléments pour prolonger l'extrémale.

Remarque : Il est important de souligner que la réfraction de l'extrémale est toujours possible et que, plus précisément dans le plan $(q, \mathrm{H})$, les

(1) Un «point» est défini par une cote d'estuaire et par les valeurs de $\mu$ et $\lambda$ qui caractérisent la marche de l'usine. 


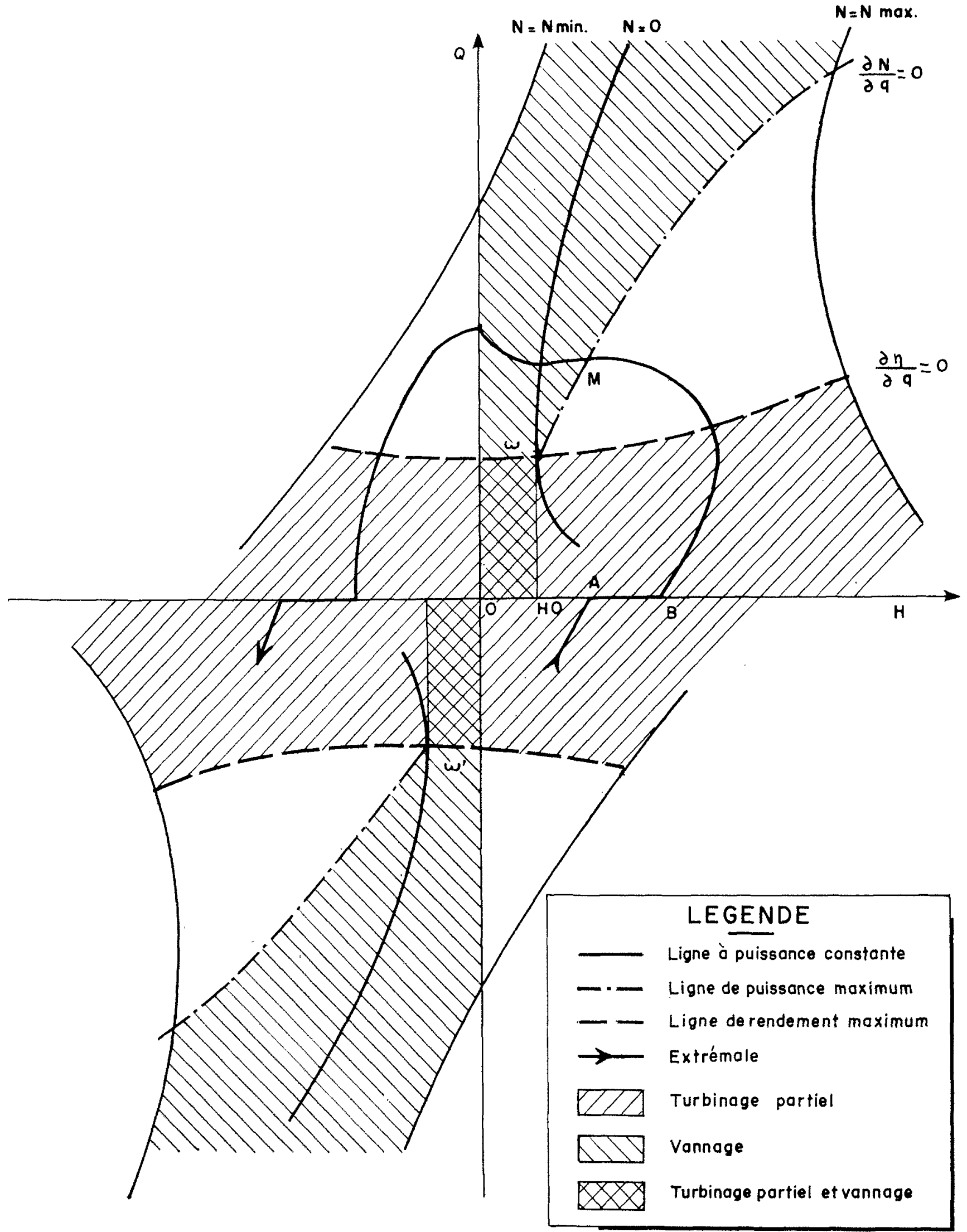

Schéma de la colline turbine. 
deux arcs se raccordent tangentiellement. Il suffit pour s'en assurer, de calculer les paramètres directeurs de la tangente.

\section{Fin du vannage.}

Dans les cycles de double effet, on atteint en général une chute nulle en marche $\left(1^{\prime}\right)$. Il est bien évident que l'on doit arrêter le vannage pour les chutes de signe opposé.

La difficulté de cette transition vient du fait que la dérivée du débit par rapport à la chute augmente indéfiniment pour une chute tendant vers zéro.

De façon plus concrète, on admet en général la formule :

$$
v_{0}=k \sqrt{\mathrm{H}}
$$

Or, les équations d'Euler font intervenir :

$$
\partial v_{0} / \partial \mathbf{H}
$$

Jusqu’à présent, en calcul numérique, on linéarisait cette loi de débit en dessous d'une chute minimale, sans se rendre compte très exactement de l'approximation faite. On peut montrer sans difficulté que la loi du débit des turbines suit asymptotiquement une loi de la même forme.

Pour simplifier l'exposé, nous supposerons $\lambda=1$ et le tarif constant. Notre problème est déterminé par la seule fonction inconnue $z$. La dérivée variationnelle s'écrit alors :

$$
\left[\mathbf{N}_{v}\right]_{z}=\frac{\partial \mathrm{N}}{\partial \mathrm{H}}-\frac{\partial \mathrm{N}}{\partial q} \frac{d v_{0}}{d \mathrm{H}}+\mathrm{S} \frac{d}{d t}\left(\frac{\partial \mathrm{N}}{\partial q}\right)=0
$$

soit :

$$
\begin{aligned}
\text { IV }\left[N_{v}\right]_{i}=\mathrm{S} \frac{\partial^{2} \mathrm{~N}}{\partial q^{2}} \dot{q}+\frac{\partial^{2} \mathrm{~N}}{\partial q \partial \mathrm{H}} \dot{\mathrm{H}}+\frac{\partial \mathrm{N}}{\partial \mathrm{H}} \\
-\frac{\mathrm{K}}{2 \sqrt{\mathrm{H}}} \frac{\partial \mathrm{N}}{\partial q}=0
\end{aligned}
$$

Or, les dérivées partielles de $\mathrm{N}$ par rapport à $q$ et $H$ sont des caractéristiques continues de la colline turbine. En première approximation, nous pouvons négliger leurs variations.

Si, lorsque $H$ devient infiniment petit, $\dot{z}$ était infiniment petit d'ordre différent de $(\mathbf{H})^{1 / 2}, \dot{q}$ serait d'ordre différent de $(H)^{-1 / 2}$ et l'équation IV ne serait pas vérifiée.

Il en résulte que $H$ et $\dot{h}$ sont équivalents et que :

$$
\dot{q} \sim \frac{k}{2 \mathrm{~S}} \cdot \frac{\partial \mathrm{N} / \partial q}{\partial^{2} \mathrm{~N} / \partial q^{2}} \cdot \frac{1}{\sqrt{\mathrm{H}}}=\frac{\mathrm{K}}{2 \sqrt{\mathrm{H}}}
$$

Le débit des vannes suit donc asymptotiquement la loi :

$$
q=q_{0}+\frac{\mathrm{K}}{\dot{h}}(\mathrm{H})^{1 / 2}
$$

\section{III. - DÉMARRAGE ET ARRET DES GROUPES}

L'équation III (2) se développe en :

$$
p(t, \lambda \mathrm{N}) \frac{\partial[\lambda \mathrm{N}(q / \lambda, \mathrm{H})]}{\partial \lambda}=0
$$

Indépendamment de la valeur de $\mu$, elle sera vérifiée si, et seulement si, les turbines fonctionnent au point de rendement maximal sous la chute H. Soit $q$ le débit correspondant. C'est une fonction de $\mathrm{H}$ seul.

$$
\lambda=\frac{q}{q^{*}}
$$

En toute rigueur, nous devrions discuter deux cas, suivant que :

$$
\mu=0 \quad \text { ou } \mu=1
$$

Or, le cas $\mu=1$ n'est possible que pour une mise en route ou un arrêt des turbines sous une chute comprise entre zéro et la chute minimale turbinale. Les conclusions relatives à ce cas peu fréquent n'apportant pas d'éléments nouveaux, et les équations afférentes étant d'un maniement lourd, nous nous contenterons de discuter les conditions de mise en route ou d'arrêt des turbines en l'absence de vannage.

Si le tarif est indépendant de la puissance, nous retrouvons les résultats antérieurs dus à M. Gibrat. Il n'y a pas de solution continue en $\lambda$. Comme pour les vannes, les turbines doivent être soit toutes en fonction, soit toutes arrêtées.

Cela devient inexact lorsque le tarif varie en fonction de la puissance. Et c'est ce point dont nous discuterons. 
Pour simplifier nous supposerons que le tarif est seulement en fonction de la puissance.

L'équation III (3), compte tenu de ces conditions, peut s'écrire (2):

$$
V \quad \frac{d p(t, \lambda N)}{d t} \mathrm{~N}\left(q^{*}, \mathrm{H}\right)=p(t, \lambda \mathrm{N}) \frac{d h}{d t} \frac{\partial \mathrm{N}}{\partial \mathrm{H}}
$$

Démarrage des groupes $[(0)-(1)$ ou $(0)-(2)]$.

Dans le cas d'un arrêt, la condition de transversalité peut se ramener à la condition :

$$
\int_{0}^{\lambda x} x \frac{d p}{d x} d x=0
$$

Si $(d p / d x)=0$ ou $\mathrm{N}=0$, l'équation $\mathrm{V}$ ne peut pas être satisfaite.

Par contre, $\lambda=0$ est une solution acceptable. L'inégalité $\lambda \geqslant 0$ impose au démarrage $\lambda \geqslant 0$.

L'équation $V$ définit $\lambda$ et cette condition devient :

$$
\dot{h} \frac{p}{p^{\prime}} \frac{\partial \mathrm{N}}{\partial \mathrm{H}} \geqslant 0
$$

Pour fixer les idées, admettons un tarif marginal dégressif; nous devons avoir :

$$
\grave{h} \frac{\partial \mathrm{N}}{\partial \mathrm{H}}<0
$$

$\partial \mathrm{N} / \partial \mathrm{H}$ ayant des signes différents sur chaque demi-colline, en tout point d'une transversale d'arrêt passe une extrémale et une seule. Elle est contenue dans la demi-colline $\mathrm{N}_{2} \quad(q<0)$ à marée montante et dans la demi-colline $\mathrm{N}_{1}$ : $(q>0)$ à marée descendante.

Ce résultat est très important. Les conclusions sont évidemment inverses pour un arrêt.

\begin{tabular}{|c|c|c|}
\hline & Démarrage & Arrêt \\
\hline$\frac{d h}{d t}>0 \ldots \ldots$ & 1 & 2 \\
\hline$\frac{d h}{d t}<0 \ldots \ldots$ & 2 & 1 \\
\hline
\end{tabular}

(2) Pour démontrer cette formule, une intégration par parties est nécessaire. Elle n'est donc valable que pour les solutions en $z$ admettant «presque partout» une dérivée seconde continue.

\section{Corollaire.}

Il y a au plus un palier par intervalle crêtecreux. Cela est évident, puisque les deux nappes de la colline sont sans communication.

\section{Délais de mise en route.}

Cette solution ne reste valable qu'autant que $\lambda$ est $<1$. A un moment donné, l'extrémal atteint la frontière $\lambda=1$ (sinon la Rance serait sûrement suréquipée). Le prolongement de l'extrémal sur cette frontière est bien connu.

La considération d'une mise en route progressive n'a d'intérêt pratique qu'autant que les délais mis en œuvre sont appréciables.

En admettant un tarif variant du quart entre 0 et $10000 \mathrm{~kW}$, nous trouvons :

Si :

$\dot{h}=2,40$ mètres/heure (coeff. 120);

$\mathrm{H}=5,50$ mètres $t=13$ minutes;

$\mathrm{H}=6,50$ mètres $t=19$ minutes;

Si :

$\dot{h}=1,50$ mètre/heure (coeff. 75);

$\mathrm{H}=3$ mètres $t=5$ minutes;

$\mathrm{H}=4$ mètres $t=12$ minutes;

$\mathrm{H}=5$ mètres $t=22$ minutes.

Ces ordres de grandeur montrent que cette branche d'extrémale peut avoir de l'influence sur la recette, donc avoir un intérêt pratique.

\section{Stabilité des arrêts.}

Recherchons à quelles conditions un arrêt $(A, B)$ est optimal par rapport à tout trajet composite formé des marches (0), (1) et (2).

D’après ce que nous avons vu antérieurement, on ne peut remplacer l'arrêt que par un trajet comportant un seul arrêt; donc, soit par. un trajet $(1)-(0)-(2)$, soit par un trajet :

$$
\text { (2) - (0) - (1) et d'extrémité (A, B). }
$$

Si nous admettons avec $M$. Caseau que, sur les lignes de rendement maximal des deux nappes de la colline turbine, les fonctions :

$$
\frac{N_{1}}{q^{\star}{ }_{1}} \text { et } \frac{N_{2}}{q^{\star}{ }_{2}} \text { sont monotones }
$$


et que les courbes :

$\frac{N_{1}}{q^{\star}{ }_{1}}$ et $-\frac{N_{2}}{q^{\star}{ }_{2}}$ ne se coupent pas,

la condition pour que l'arrêt soit optimal entre (A, B) par rapport aux trajets voisins permis prend la forme extrêmement élégante :

$$
\left.\left(\frac{\mathrm{N}}{q^{\star}}\right)_{\mathrm{A}}=-\frac{\mathrm{N}}{q^{*}}\right)_{\mathrm{B}}
$$

Ce résultat capital nous permet de prolonger une extrémale ayant atteint un arrêt, au-delà de celui-ci.

\title{
CONCLUSION
}

Cette étude rapide nous a montré que les conditions de réflexion ou de réfraction d'une extrémale donnée sont toujours déterminées, même pour un tarif fonction de la puissance.
Ce qui revient à dire que l'ensemble des extrémales en double effet issues d'une cote donnée ne dépend que d'un seul paramètre continu. Ce résultat global permet de mieux cerner le problème de l'optimum global.

\section{IMPORTANT BUREAU D'ÉTUDES}

recherche :

\section{JEUNE INGÉNIEUR HYDRAULICIEN}

\author{
ayant expérience Hydraulique agricole \\ pour poste PARIS comportant missions OUTRE-MER
}

Ecrire à B. 306, PUBLIPRESS, 31, bd Bonne-Nouvelle, Paris $\left(2^{\circ}\right)$ qui transmettra. 